\title{
Density profile and line-of-sight mass contamination of SLACS gravitational lenses
}

\author{
C. C. Guimarães and Laerte Sodré Jr.
}

Departamento de Astronomia, IAG, Universidade de São Paulo

We use data from 58 strong lensing events surveyed by the Sloan Lens ACS Survey to estimate the projected galaxy mass inside their Einstein radii by two independent methods: stellar dynamics and strong gravitational lensing. We perform a joint analysis of both estimates examining the galaxy-lens density profile (that we approximate by a power law), the anisotropy of the velocity distribution (represented by an effective constant parameter), and a possible line-of-sigh (l.o.s.) mass contamination (which is suggested by various independent works in the literature). For each model, a likelihood analysis is performed to find the parameters that produce the best agreement between the dynamical and lensing masses, and the parameter confidence levels. The Bayesian evidence is calculated to allow a comparison among the models. We find a degeneracy among the slope of the density profile, the anisotropy parameter and the l.o.s. mass contamination. For a density profile close to isothermal, a l.o.s. mass contamination of the order of a few percent is possible, being less probable with larger anisotropy.

\section{References}

Guimarães, A. C. C. \& Sodré, L. J. 2009, arXiv:0904.4381 